\title{
Value of the swimming position and arm traction in visualizing the cervicothoracic junction over the standard lateral cervical X-ray
}

\author{
Aydin Toksoy • Firat Bektas • Cenker Eken • \\ Kaan Ceken - Yildiray Cete
}

Received: 13 May 2009 /Accepted: 11 January 2010/Published online: 23 March 2010

(C) Springer-Verlag London Ltd 2010

\begin{abstract}
Background The cervicothoracic junction (CTJ) is often inadequately visualized on lateral cervical X-rays due to anatomic variations and technical factors.

Aims The aim of this study was to investigate whether the swimmer's view and arm traction could enhance the image field on the standard lateral cervical (SLC) X-ray.

Methods The study was conducted in a university hospital in October 2007 with 40 volunteers. SLC X-ray, lateral cervical X-ray in the swimming position, and lateral cervical X-ray with arm traction were performed in the supine position. The enhancements in the image fields were analyzed.

Results There was a statistically significant difference for the increases in the view of cervical spines between SLC X-ray $(12.60 \pm 7.48)$ and either lateral cervical X-ray with arm traction $(21.73 \pm 9.78 ; p=0.000)$ or in the swimming position $(21.20 \pm 14.19 ; p=0.001)$. Both arm traction and swimming position increased the field of view by approximately $9 \mathrm{~mm}$. Increased visualization of the cervical spine occurred for 24
\end{abstract}

The views expressed in this paper are those of the author(s) and not those of the editors, editorial board or publisher.

\footnotetext{
A. Toksoy $\cdot$ F. Bektas $\cdot$ C. Eken $(\bowtie) \cdot$ Y. Cete

Department of Emergency Medicine,

Akdeniz University Medical Faculty,

07059 Antalya, Turkey

e-mail: cenkereken@akdeniz.edu.tr

F. Bektas

e-mail: fbektas@akdeniz.edu.tr

Y. Cete

e-mail: ycete@akdeniz.edu.tr

K. Ceken

Department of Radiology, Akdeniz University Medical Faculty,

07059 Antalya, Turkey
}

of the 40 participants using the arm traction view (60.0\%) and 23 participants $(57.5 \%)$ using the swimming position view - results found to be statistically similar according to the $\geq 1 / 3$ caudal vertebral height visualized $(p=0.902)$. Using the lateral cervical X-ray view, the number of cervical vertebrae visualized differed according to body mass index (BMI) - seven cervical vertebrae were visualized in participants with a $\mathrm{BMI}<25$ and six vertebrae were visualized in participants with a $\mathrm{BMI} \geq 25(p=0.007)$.

Conclusion Lateral cervical X-rays with arm traction and swimming position enhance the view of SLC X-rays. An initial SLC X-ray including the lower third of the cervical spine (with $\mathrm{C} 7$ ), arm traction, and swimming position may be beneficial in visualizing the CTJ. However, patients with an increased BMI are unlikely to benefit from all three methods.

Keywords Lateral cervical X-ray · Arm traction · Swimming position $\cdot$ Body mass index $\cdot$ Trauma

\section{Introduction}

For patients presenting with multiple trauma to the emergency department (ED), advanced trauma life support (ATLS) guidelines recommend that lateral and anteroposterior cervical $\mathrm{X}$-rays should demonstrate a complete visualization of: (1) all cervical vertebrae, (2) the cervicothoracic junction (CTJ), and (3) the first thoracic vertebra [1]. The misdiagnosis of cervical spine injury may have terrible consequences, particularly in the cervicothoracic region, where $9-18 \%$ of cervical spine injuries are seen [2-4].

However, the CTJ is often inadequately visualized on lateral cervical $\mathrm{X}$-rays due to anatomic variations and technical factors. Body mass index (BMI) and the length of 




Fig. 1 Position of the study participants and placement of X-ray source and film cartridge during SLC X-ray

the neck and shoulders are the major anatomic factors leading to insufficient view of the CTJ $[5,6]$. Traditionally, the swimmer's view or arm traction is used in these cases [1]. Physicians also use the oblique view in clinical practice when the standard view is insufficient. Computed tomography (CT) and magnetic resonance imaging (MRI) are ultimately used in the cases in which X-rays have failed [7-9].

The aim of this study was to investigate whether the swimmer's view and arm traction could enhance the image field on the standard lateral cervical (SLC) X-ray.

\section{Materials and methods}

Study design

This study is a comparison of the SLC view with the arm traction view and swimming position view. The study was conducted in a university hospital in October 2007. The study population comprised volunteers from the ED staff, including physicians, nurses, and paramedics. Informed consent was taken from all volunteers. The local Ethics Committee approved this study. The exclusion criteria were pregnancy or suspicion of pregnancy, a history of cervical spinal trauma or operation, and refusing to give informed consent.

\section{Data collection}

Initially, demographics, body weight and height, length of the neck, and the distance between the two shoulders were recorded for each volunteer. Sternomental distance (SMD) was used in order to determine the length of the neck. The distance between two acromions-biacromial distance (BAD) - served as a measure of the distance between two shoulders.

\section{Processing}

SLC X-ray, lateral cervical X-ray in the swimming position, and lateral cervical X-ray with arm traction were performed in the supine position for each volunteer. The supine position was preferred because the typical trauma patients will usually undergo $\mathrm{X}$-ray in the supine position instead of standing up.
Fig. 2 Position for the lateral cervical X-ray with traction of both arms

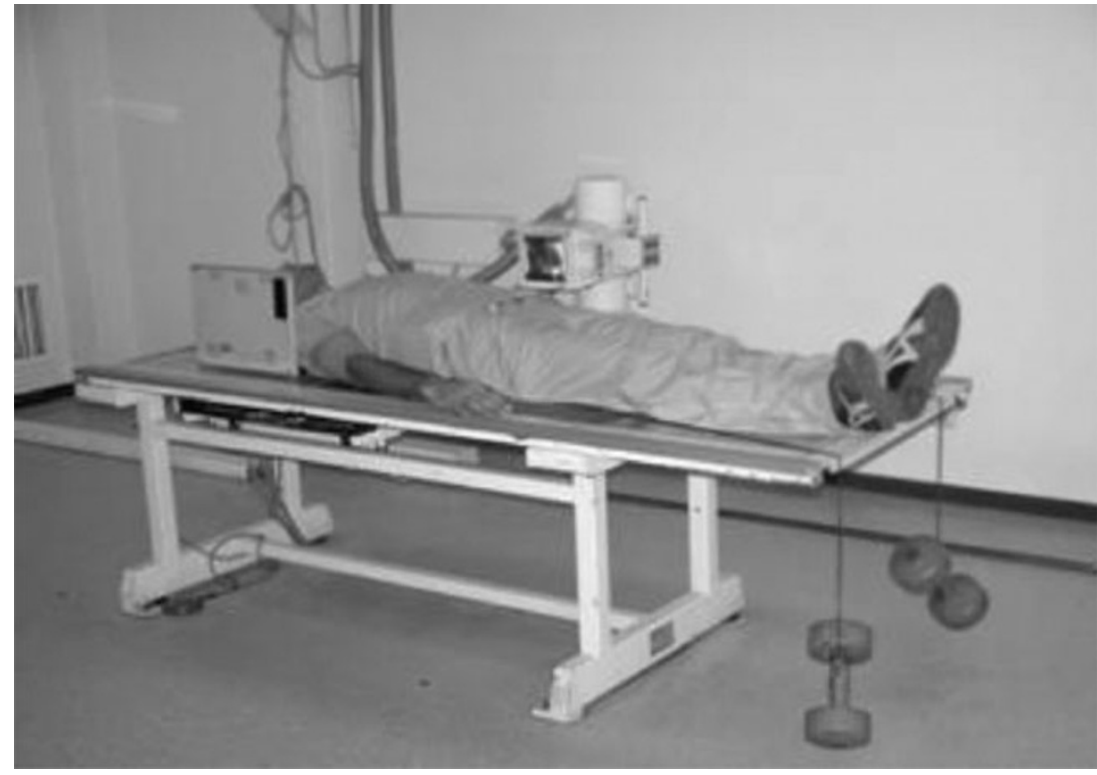


Fig. 3 Position for the lateral cervical X-ray taken in the swimming position

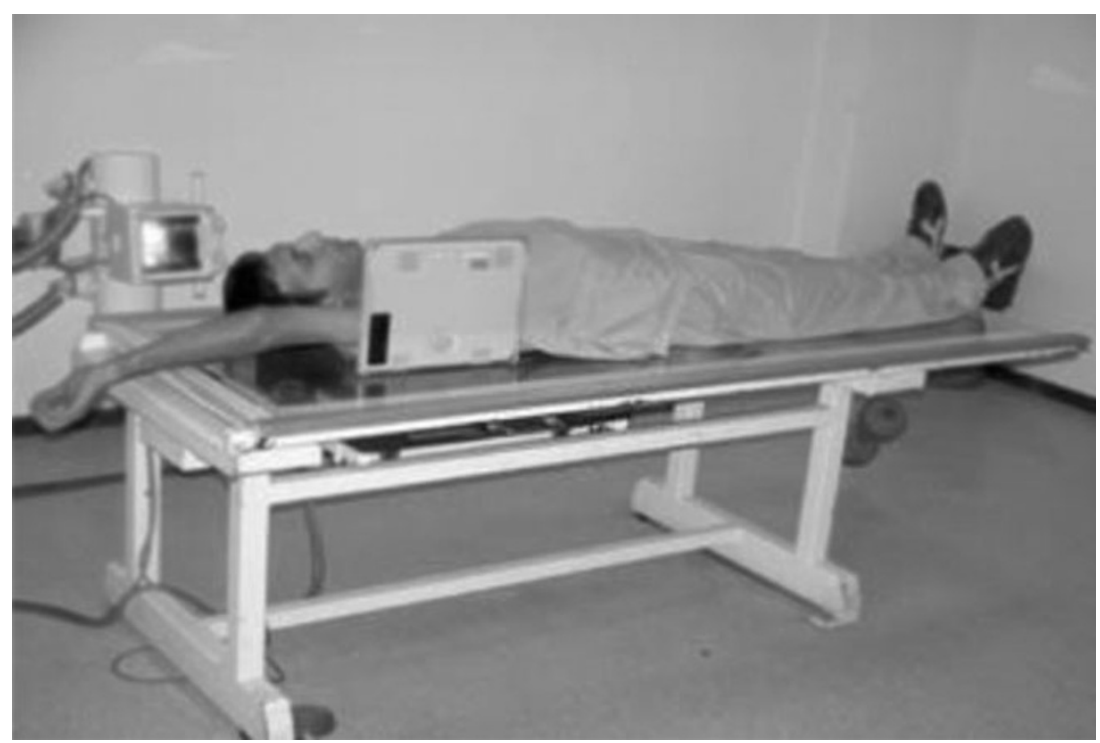

Cervical X-rays were taken by a radiology technician with 20 years of experience. An assistant professor of radiology and the research assistant from our ED accompanied him. Study participants were placed on the X-ray table in a supine position with complete contact of the head with the X-ray table. The X-ray film cartridge was placed to the right side of the participant's head and at $4 \mathrm{~cm}$ superior to the ear pinnacle. The central X-ray source was located perpendicularly to the film cartridge at the approximate level of the fourth cervical vertebra. The SLC X-rays used a standard dose of radiation within a time interval of 0.08 $0.12 \mathrm{~ms}$, using $65-70 \mathrm{~kW}$ of voltage and a current of 100 $\mathrm{mA}$. The position of the study participants and placement of $\mathrm{X}$-ray source and film cartridge during the SLC X-ray are shown in Fig. 1.

\section{Lateral cervical X-ray with arm traction}

Four kilograms of weight were fixed onto both arms over the elbows. The weights were hung down the end point of the X-ray table through the feet of the patient (Fig. 2). The $\mathrm{X}$-ray machine and film cartridge were placed similarly to the SLC X-ray and the same doses were used. We believed that the weight placed over the elbows would be effective in overcoming the opposing resistance from voluntary muscle contraction.

\section{Lateral cervical X-ray in the swimming position}

Patients were placed in the supine position with the right arm placed overhead with full extension of the shoulder and the left arm undergoing traction caudally. The film cartridge was placed under the axilla on the right side with the superior edge of the cartridge located $4 \mathrm{~cm}$ superior to the ear pinnacle. The X-ray source focused on the CTJ (level between the seventh cervical vertebra and first thoracic vertebra). A standard dose of radiation was used, within a time interval of $0.12-0.16 \mathrm{~ms}$, using $65-75 \mathrm{~kW}$ of voltage and a current of $150 \mathrm{~mA}$. Figure 3 shows the position for the lateral cervical X-ray taken in the swimming position.

All X-rays were taken with the Toshiba TF-6-TL-6 machine from $100 \mathrm{~cm}$ distance using cartridges with a size of $24 \times 30 \mathrm{~cm}$.

\section{Outcome measures}

Lateral cervical spine X-rays were performed in all subjects with the SLC view, arm traction view, and swimming position view, with the heights of the lowest vertebra measured in each case. The vertical height of the SLC view was then compared with that of the arm traction and swimming position views. The lowest point seen in the X-rays constituted by the superior edges of the clavicle or soft tissue was accepted as the reference point for all three views.

Table 1 Demographics of study participants

\begin{tabular}{lll}
\hline Variable & Mean \pm SD & Median (min.-max.) \\
\hline Age & $30 \pm 4.9$ & $30(20-44)$ \\
Gender, male/female (\%) & $24(60) / 16(40)$ & \\
Weight & $71.25 \pm 16.82$ & $70(45-110)$ \\
Height & $168.55 \pm 8.93$ & $170(148-187)$ \\
BMI & $24.87 \pm 4.52$ & $24.5(18-36.3)$ \\
SMD & $10.83 \pm 1.58$ & $11(8-14)$ \\
BAD & $40.98 \pm 3.18$ & $41.5(34-46)$ \\
\hline
\end{tabular}

$S D$ standard deviation, $B M I$ body mass index, $S M D$ sternomental distance, $B A D$ biacromial distance, min. minimum, max. maximum 
Table 2 Enhancement in the view of cervical spines after arm traction and swimming position

\begin{tabular}{lll}
\hline Lateral cervical X-rays & Mean $\pm \mathrm{SD}(\mathrm{mm})$ & $p$ value \\
\hline Standard & $12.60 \pm 7.48$ & \\
Arm traction & $21.73 \pm 9.78$ & $0.000^{\mathrm{a}}$ \\
Swimming position & $21.20 \pm 14.19$ & $0.001^{\mathrm{b}}$ \\
$p$ value & $0.841^{\mathrm{c}}$ & \\
\hline
\end{tabular}

${ }^{\mathrm{a}}$ Comparison of SLC X-ray and lateral cervical X-ray with traction

${ }^{\mathrm{b}}$ Comparison of SLC X-ray and lateral cervical X-ray with swimming position

${ }^{\mathrm{c}}$ Comparison of the enhancement with traction and swimming position

Patients were also categorized according to whether an enhanced view of the cervical spine was obtained-with visualization of more than one third of the lowest cervical vertebral body. If the visualization was less than this height, the view was considered not enhanced.

All of these X-ray evaluations and measurements were performed by a radiologist aware of the study but blinded to study subjects.

\section{Statistical analysis}

The study data were analyzed in SPSS 10.0 for Windows. Continuous variables were expressed as mean \pm standard deviation and ordinal variables expressed as median and minimum-maximum. Comparisons of two groups with normal distribution were performed by Student' $t$ test. The Mann-Whitney U test was used for two group comparisons without normal distribution. Related samples with normal distribution for two groups were analyzed by the related $t$ test and dichotomized variables with the McNemar test. Normal distribution was tested by the KolmogorovSmirnov test for sample sizes over 30 and Shapiro-Wilk if sample sizes were under 30. All of the hypotheses were constructed as two-tailed and an alpha value of 0.05 was accepted as significant.

\section{Results}

The study population was composed of 40 volunteers. The mean age of the participants was $30 \pm 4.9$ with a range of 20-44 years and $60 \%(n=24)$ of them were male. The weight, height, BMI, SMD, and BAD of the participants are displayed in Table 1.

There was a statistically significant difference for the increases in the view of cervical spines between SLC X-ray $(12.60 \pm 7.48)$ and either lateral cervical X-ray with arm traction $(21.73 \pm 9.78 ; p=0.000)$ or in the swimming position $(21.20 \pm 14.19 ; p=0.001)$ (Table 2). Both arm traction and swimming position increased the field of view by approximately $9 \mathrm{~mm}$. Direct comparison of increased vertical height between the arm traction and swimming position views showed no difference in increased visualization $(p=0.841)$.

Increased visualization of the cervical spine occurred for 24 of the 40 participants using the arm traction view $(60.0 \%)$ and 23 participants $(57.5 \%)$ using the swimming position view - results found to be statistically similar ( $p=$ 0.902) (Table 3).

For the arm traction and swimming position views, there was also no difference between groups showing or not showing an enhanced view-defined earlier in this paper as visualization of more than one third of the lowest cervical vertebral body - according to age, gender, BMI, SMD, and BAD (Table 4).

Fifty percent of participants had a normal BMI (BMI< $25)$. Using the SLC view, the number of cervical vertebrae visualized differed according to $\mathrm{BMI}$ - seven cervical vertebrae were visualized in participants with $\mathrm{BMI}<25$ and six vertebrae were visualized in participants with a $\mathrm{BMI} \geq 25(p=0.007)$ (Table 5).

The caudal endpoint for vertebral height for this study was either soft tissue opacity or the clavicle. However, vertebral bodies below the clavicle were visualized in 15 patients $(37.5 \%)$ using the swimming position view, with over $70 \%(11 / 15)$ of these participants having a normal BMI. No images showed vertebral bodies below the

Table 3 Proportions of patients classified as having an enhanced view with arm traction and swimming position

\begin{tabular}{|c|c|c|c|c|c|}
\hline & & \multicolumn{2}{|l|}{ Swimming position } & \multirow[t]{2}{*}{ Total } & \multirow[t]{2}{*}{$p$ value } \\
\hline & & $\begin{array}{l}\text { Patients with no } \\
\text { enhancement }(\geq 1 / 3 \text { caudal } \\
\text { vertebral height visualized) }\end{array}$ & $\begin{array}{l}\text { Patients with enhancement } \\
\text { ( } \geq 1 / 3 \text { caudal vertebral height visualized) }\end{array}$ & & \\
\hline \multirow[t]{2}{*}{ Arm traction } & Patients with enhancement & $10(25 \%)$ & $6(15 \%)$ & $16(40 \%)$ & 0.902 \\
\hline & Patients with enhancement & $7(17.5 \%)$ & $17(42.5 \%)$ & $24(60 \%)$ & \\
\hline Total & $17(42.5 \%)$ & $23(57.5 \%)$ & $40(100 \%)$ & & \\
\hline
\end{tabular}


Table 4 Comparison of patients with and without enhanced ( $\geq 1 / 3$ caudal vertebral height visualized) view after arm traction and swimming position

\begin{tabular}{|c|c|c|c|c|c|c|}
\hline \multirow[t]{2}{*}{ Variable } & \multicolumn{2}{|c|}{ Lateral cervical X-ray with traction } & \multirow[t]{2}{*}{$p$} & \multicolumn{2}{|c|}{ Lateral cervical X-ray with swimming position } & \multirow[t]{2}{*}{$p$} \\
\hline & $\begin{array}{l}\text { Patients with no } \\
\text { enhancement }\end{array}$ & $\begin{array}{l}\text { Patients with } \\
\text { enhancement }\end{array}$ & & $\begin{array}{l}\text { Patients with no } \\
\text { enhancement }\end{array}$ & $\begin{array}{l}\text { Patients with } \\
\text { enhancement }\end{array}$ & \\
\hline Age & $29.9 \pm 5$ & $30 \pm 4.9$ & 0.672 & $32 \pm 5.5$ & $28.5 \pm 3.9$ & 0.055 \\
\hline Gender male $(\%)$ & 56.3 & 62.5 & 0.693 & 64.7 & 56.5 & 0.601 \\
\hline BMI & $23.9 \pm 4.9$ & $25.5 \pm 4.2$ & 0.296 & $24 \pm 3.7$ & $25.5 \pm 5$ & 0.343 \\
\hline SMD & $10.94 \pm 1.57$ & $10.75 \pm 1.62$ & 0.719 & $11.24 \pm 1.64$ & $10.52 \pm 1.5$ & 0.162 \\
\hline BAD & $40.38 \pm 3.44$ & $41.4 \pm 3$ & 0.337 & $41.29 \pm 2.89$ & $40.74 \pm 3.43$ & 0.592 \\
\hline
\end{tabular}

$S D$ standard deviation, $B M I$ body mass index, $S M D$ sternomental distance, $B A D$ biacromial distance

clavicle in participants using the arm traction view. Posterior structures could be visualized in all arm traction images and in $35 \%$ (14/40) of swimming position images.

\section{Discussion}

Visualizing the CTJ by SLC X-ray remains a challenge in trauma patients. Lateral cervical X-rays with arm traction and swimming position are suggested by this study as an effective alternative to visualize the CTJ in these patients. Our results demonstrate that both arm traction and swimming position views have been found to increase visualization of the cervical spine by an average of $9 \mathrm{~mm}$. Both views have also been shown to increase visualization of more than one third of the caudal vertebral body in more than half of the participants.

While both arm traction and swimming position views hold benefit with better visualization, each view has been shown in our study to have unique advantages. The swimming view achieves visualization below the clavicle in some participants $(38 \%)$. This view also visualized posterior structures - crucial in the evaluation of trauma patients - in roughly the same proportion of participants $(35 \%)$. However, the arm traction view appears to be much more effective in visualizing the posterior structures, as it did in all participants in our study.

BMI does not restrict the advantages of both methods according to the results of this study. However, BMI was revealed as a limiting factor in the image field of SLC Xray. The median cervical spine level seen in SLC is the sixth vertebra in patients with high BMI and seventh in patients with normal BMI. However, only $9 \mathrm{~mm}$ of additional vertical height is added with the use of arm traction or the swimming position. In this situation, all three methods, SLC X-ray, arm traction, and swimming position, are likely to be insufficient in patients with high BMI who present to the ED with trauma. CT or MRI should be alternative imaging methods in these patients as an initial choice. And also the vertebral bodies below the clavicle are more likely to be seen during the swimming position in normal patients than in patients with high BMI.

Previous evidence in the literature is somewhat limited and results varied. A few studies observed the effects of arm traction on imaging of the cervical spine. Bowe et al. found that traction enhances the view of the cervical spine for more than two thirds of a vertebral body [10]. Ohiorenoya et al. reported that unless an initial cervical spine radiograph includes the upper one third of the body of the seventh cervical vertebra, the probability of attaining the $\mathrm{C} 7 / \mathrm{T} 1$ level with arm traction is $<15 \%$ [11]. Our results approximate the results of a previous study by Ireland et al., which reported that $37 \%$ of patients imaged with the swimming position view showed visualization of the posterior structures [6]. A recent retrospective study by Rethnam et al. showed inadequacy of the swimming position in visualizing the CTJ [14]. However, their study did not evaluate the possible factors affecting the view of these techniques. And the basic view of patients obtained with SLC X-ray had not been regarded in their study.

Traction techniques have also been observed in the literature. Bowe et al. compared different traction techni-
Table 5 Number of cervical vertebrae visualized on SLC Xray according to the BMI

$B M I$ body mass index

\begin{tabular}{llllllllllllll}
\hline \multicolumn{10}{c}{} & \multicolumn{1}{c}{ Number of cervical vertebrae visualized on SLC X-ray } \\
\cline { 2 - 11 } & BMI & C1 & C2 & C3 & C4 & C5 & C6 & C7 & T1 & Median & $p$ \\
\hline Number of patients & $<25$ & 0 & 0 & 0 & 0 & 1 & 8 & 10 & 1 & 7 & 0.007 \\
& $\geq 25$ & 0 & 1 & 0 & 3 & 5 & 6 & 5 & 0 & 6 & \\
\hline
\end{tabular}


ques to SLC X-ray [10] and found improved visualization with manual traction alone and accompanied by inspiration and expiration. Interestingly, the study also found that traction using weights of over $18 \mathrm{~kg}$ did not improve visualization using the arm traction view. Boger and Ralls described an auto-traction device for radiography of the lower cervical spine. They used a belt attached to the wrist of patients and patients pulled this belt with their feet [12]. Norris et al. compared a traction device to manual traction [13]. Arm traction with weights used in our study showed benefit in increasing visualization. This technique also holds additional benefit, as it may lower the burdens of time spent and radiation exposure inherent in providing manual traction for trauma patients.

Another finding of this study is that the length of the neck and the size of the shoulders do not affect the results of lateral cervical X-rays taken with arm traction and in the swimming position.

\section{Limitations}

There are some limitations to this study. The sample size is small and should be increased for more accurate subgroup analyses. Another limitation is that participants in our study did not use a cervical collar. However, patients who present to the ED needing cervical spine imaging are often the victims of trauma, who typically have a cervical collar placed when they present. Further study should observe patients wearing a cervical collar.

\section{Conclusion}

Lateral cervical X-rays with arm traction and swimming position enhance the view of SLC X-rays. An initial SLC $\mathrm{X}$-ray including the lower third of the cervical spine (with C7), arm traction, and swimming position may be beneficial in visualizing the CTJ. However, patients with an increased BMI are unlikely to benefit from all three methods. The swimming position offered some visualization of the posterior structures and thoracic vertebral bodies in some participants. Arm traction demonstrated visualization of posterior structures of all participants imaged.
Acknowledgement This study was supported by Akdeniz University Foundation. And great thanks to Dr. Joshua Schiller for his contributions to the article.

\section{References}

1. American College of Surgeons Committee on Trauma (2004) Advanced trauma life support, 7th edn. Spine and spinal cord trauma, pp 178-204

2. Miller MD, Gehweiler JA, Martinez S, Charlton OP, Daffner RH (1978) Significant new observations on cervical spine trauma. AJR Am J Roentgenol 130:659-663

3. Nichols CG, Young DH, Schiller WR (1987) Evaluation of cervicothoracic junction injury. Ann Emerg Med 16:640-642

4. Gisbert VL, Hollerman JJ, Ney AL et al (1989) Incidence and diagnosis of C7-T1 fractures and subluxations in multiple-trauma patients: evaluation of the advanced trauma life support guidelines. Surgery 106:702-709

5. Amin A, Saifuddin A (2005) Fractures and dislocations of the cervicothoracic junction. J Spinal Disord Tech 18:499-505

6. Ireland AJ, Britton I, Forrester AW (1998) Do supine oblique views provide better imaging of the cervicothoracic junction than swimmer's views? J Accid Emerg Med 15:151-154

7. Hoffman JR, Mower WR, Wolfson AB, Todd KH, Zucker MI (2000) Validity of a set of clinical criteria to rule out injury to the cervical spine in patients with blunt trauma. National Emergency X-radiography Utilization Study Group. N Engl J Med 343:94-99

8. Jenkins MG, Curran P, Rocke LG (1999) Where do we go after the three standard cervical spine views in the conscious trauma patient? A survey. Eur J Emerg Med 6(3):215-217

9. Wang VY, Chou D (2007) The cervicothoracic junction. Neurosurg Clin N Am 18:365-371

10. Bowe CT, Burton JH, Chandler RC, Davidson RM (2003) The effect of arm traction on the adequacy of cross-table lateral cervical spine radiographs. J Trauma 55:800-801

11. Ohiorenoya D, Hilton M, Oakland CD, McLauchlan CA, Cobby M, Hughes AO (1996) Cervical spine imaging in trauma patients: a simple scheme of rationalising arm traction using zonal divisions of the vertebral bodies. J Accid Emerg Med 13:175-176

12. Boger D, Ralls P (1981) New traction device for radiography of the lower cervical spine. AJR Am J Roentgenol 137:1202-1204

13. Norris CS, Silva WE, Dodson TF, Darrow J (1986) Shoulder harness traction for roentgenographic assessment of the cervical spine. Arch Surg 121:452-455

14. Rethnam U, Yesupalan RS, Bastawrous SS (2008) The swimmer's view: does it really show what it is supposed to show? A retrospective study. BMC Med Imaging 15:2

Cenker Eken is 34 years old and has been working in the Emergency Department of Akdeniz University Medical Faculty, in Turkey, as an assistant professor. He started his residency program in 2000 and finished it in 2004. He is particularly interested in research, biostatistics, and evidence-based medicine. 